\title{
Efficacy and safety of oral corticosteroids and olfactory training in the management of COVID-19-related loss of smell
}

\author{
Serge-Daniel Le Bon ${ }^{1}$ ( ) Deborah Konopnicki ${ }^{2}$. Nathalie Pisarski ${ }^{1}$. Léa Prunier ${ }^{1}$ Jérôme R. Lechien ${ }^{1}$. \\ Mihaela Horoi ${ }^{1}$
}

Received: 29 October 2020 / Accepted: 25 November 2020 / Published online: 9 January 2021

○) Springer-Verlag GmbH Germany, part of Springer Nature 2021

\begin{abstract}
Purpose As the COVID-19 pandemic continues, an increasing number of patients are afflicted by olfactory loss, a now well-recognized symptom of the disease. Though many patients seem to recover their sense of smell after a few weeks, a certain proportion of them seem to develop long-lasting olfactory disorder. Yet, as of October 2020, there is no recommended standardized treatment to reduce the risk of developing long-term olfactory disorder. In this pilot study, we investigated the efficacy and the safety of oral corticosteroids and olfactory training as a treatment for patients with persistent olfactory dysfunction as a result of COVID-19.

Methods Non-hospitalized patients with a sudden loss of smell and a confirmed COVID-19 diagnosis were recruited by hospital call from February to April 2020. These participants were submitted to an extensive psychophysical testing in order to identify those with persistent dysosmia. Dysosmic patients were then treated either by a 10-day course of oral corticosteroids combined with olfactory training, or by olfactory training alone. All participants were subject to a second olfactory test after a mean of 10 weeks.

Results 72 subjects with documented COVID-19 infection performed the initial olfactory test, on average 5 weeks after losing their sense of smell. Amongst them, 27 (37.5\%) patients showed persistent dysosmia and were all included in this study. Nine participants received oral corticosteroids and performed olfactory training (OCS + OT), while 18 performed olfactory training (OT) only. Only participants in the OCS + OT group had significantly improved their olfactory score and did so above the minimal clinically important difference for subjective improvement of smell $(p=0.007)$. Three of the participants who received oral corticosteroids reported minimal and transient side effects.

Conclusion This pilot study may suggest the combination of a short course of oral corticosteroids and olfactory training is safe and may be beneficial in helping patients with enduring dysosmia recover from olfactory loss due to COVID-19. There is a crucial need for further investigation with larger cohorts to corroborate these findings.
\end{abstract}

Keywords Oral corticosteroids · Olfactory training $\cdot$ Loss of smell $\cdot$ Anosmia $\cdot$ COVID-19

\section{Introduction}

The clinical evolution of loss of smell due to COVID-19 is still unclear as reports of recovery vary significantly, ranging from 4 to $89 \%$ a month after the onset of anosmia $[1,2]$.

Serge-Daniel Le Bon

sergelebon@gmail.com

1 Department of Otorhinolaryngology, Head and Neck Surgery, CHU Saint-Pierre, Université Libre de Bruxelles (ULB), Brussels, Belgium

2 Department of Infectious Diseases, CHU Saint-Pierre, Université Libre de Bruxelles (ULB), Brussels, Belgium
Studies relying on more reliable data, such as psychophysical testing, showed persistent abnormal olfactory scores in $37 \%$ to $52 \%$ of patients 5 weeks after onset [2,3]. Loss of smell due to COVID-19 may be more prevalent and severe than in other viral upper respiratory infections. Huart et al. found that olfactory function was more affected in patients with COVID-19 than those with common cold [4]. As they pointed out, though most patients should make a full recovery, it is likely that a certain proportion of them will develop permanent post-viral olfactory dysfunction (PVOD). This is already known to be the case for patients with an upper respiratory infection caused by viruses such as parainfluenza, rhinovirus, or other coronaviruses [5]. Even a small 
proportion of affected patients may number in the tens of thousands in the case of COVID-19. Permanent olfactory loss may lead many to suffer from known comorbidities, such as depression and impaired cognition, and even maybe from earlier death $[6,7]$.

To date there is no evidence-based medical intervention to help patients with persistent dysosmia due to COVID-19, although olfactory training (OT) has been recommended [8]. Initially, oral corticosteroids (OCS) were strongly contraindicated by the World Health Organization; however, mounting evidence shows significant benefit on mortality in patients with severe forms of COVID-19 [9-11]. Moreover, some studies have shown that systemic corticosteroids actually improve olfaction in some patients with PVOD [12, 13]. However, it has been argued that this benefit may only be observed if the patient is treated at an early stage of the acute phase [14]. Currently, no studies have investigated the use of OCS and OT in a prospective cohort. In this short communication, we report a short-term evaluation of efficacy and safety of OCS and OT in patients with persisting dysosmia due to COVID-19.

\section{Material and methods}

This pilot prospective study is the continuation of our study on 72 non-hospitalized patients with loss of smell due to COVID-19 [2]. The study protocol was approved by the Review Board of our institution (CHUSP 200422). Written informed consent was obtained from all participants.

Orthonasal olfactory function was evaluated by the "Sniffin' Sticks" battery test (Burghart GmbH, Wedel, Germany) which comprises three olfactory tasks: threshold, discrimination, and identification. The sum of the scores from the three subtests makes up the "threshold discrimination identification score" (or TDI score) which was used clinically to assess olfactory performance. Participants were considered normosmic or dysosmic when TDI $\geq 30.75$ and $<30.75$, respectively [15].

The first olfactory testing session (TDI-1) was performed in April 2020, 5 weeks after the onset of loss of smell on average. Patients who scored in dysosmic levels were separated into two groups: one receiving a 10-day course of $32 \mathrm{mg}$ of methylprednisolone once daily combined with OT, and the other performing OT alone. All patients showed no persistent symptoms of COVID infection, except for olfactory loss. They were all checked for potential contraindications to OCS, such as immunosuppressive conditions (e.g., AIDS, chemotherapy), active infection, peptic ulcer, diabetes, glaucoma, psychotic disorder, or recent attenuated vaccine. Given the safety concerns about oral steroid use held by the medical community and by the general population, participants were informed about potential side effects of a short course of OCS (such as sleeplessness, sugar craving, stomach upset, blood pressure increase) and were asked to opt for treatment group on a voluntary basis. All patients performed a second olfactory testing session (TDI-2) after 10 weeks to assess the evolution of olfactory function. The change in TDI scores between both sessions is referred here as $\Delta \mathrm{TDI}=\mathrm{TDI}-2-\mathrm{TDI}-1$.

For each session of OT, patients were asked to mindfully sniff four odors for approximately $10 \mathrm{~s}$ each (rose, eucalyptus, lemon, cloves). These were provided in a 'Smell Training Kit' (Dos Medical-BV, Heteren, Netherlands). Patients were asked to perform sniffing sessions twice daily, for 10 weeks. They had to report their compliance to OT which was evaluated with the following formula: number of OT sessions per day $/ 2 \times$ number of days per week $/ 7 \times$ number of weeks/10.

Continuous variables were compared using Wilcoxon signed-rank test and Mann-Whitney test for two related and independent groups, respectively. Fisher's exact test was used to compare categorical data, and Spearman correlation to analyze the relationship between age, sex, compliance to OT, and $\Delta$ TDI score. Statistical significance was fixed at $\alpha=0.05$. All analyses were performed using the Statistical Package for the Social Sciences (SPSS version 25; IBM Corp, Armonk, NY, USA).

\section{Results}

Based on extensive olfactory psychophysical testing, 27 (37.5\%) out of 72 patients were still dysosmic 5 weeks after having lost their sense of smell (6 had anosmia, 21 had hyposmia). Only 9 participants volunteered for the OCS + OT group, whereas 18 opted for performing OT alone. As aforementioned, this mismatch in group size was mainly due to safety concerns about steroid use among study participants. Although both groups were not randomized, age, sex and initial olfactory score (TDI-1) showed no statistically significant difference (Table 1). After 10 weeks, patients in the OCS + OT group had significantly improved their olfactory score by 7.7 points on average $(p=0.007)$, compared with a 2.1-point increase in the OT group $(p=0.126)$ (Fig. 1). Additionally, a Mann-Whitney U Test confirmed the significant difference in $\Delta$ TDI scores between the OCS + OT group and the OT group $(p=0.046)$.

Average compliance to OT was 43\% (CI 23-63\%) for the OCS + OT group, and 31\% (CI 16-47\%) for the OT alone group, but this difference was not statistically significant. Neither age, sex, or compliance to OT was correlated to TDI score in any group. Of the nine participants who received OCS, three reported minimal and transient side effects such as abdominal pain and insomnia. No patient reported side effects related to olfactory training. 
Table 1 Patient characteristics, olfactory scores, and compliance to olfactory training according to treatment group in 27 patients with persistent dysosmia due to COVID-19

\begin{tabular}{lllll}
\hline & OCS + OT $(N=9)$ & OT $(N=18)$ & $p$ value & Test \\
\hline Patient characteristics & & & & \\
Age (mean \pm SD), years old & $42 \pm 14$ & $44 \pm 14$ & NS & MW \\
Sex (F/M) & $7 / 2$ & $14 / 4$ & NS & F \\
Olfactory testing & & & & \\
TDI-1 score (mean \pm SD), /48 & $19.7 \pm 9.9$ & $24.9 \pm 6.1$ & NS & MW \\
$\Delta$ TDI score (mean \pm SD), /48 & $7.7 \pm 5.1$ & $2.1 \pm 5.5$ & 0.046 & MW \\
Olfactory training & & & & \\
Compliance (mean \pm SD), $\%$ & $43 \pm 26$ & $31 \pm 31$ & NS & MW \\
\hline
\end{tabular}

$F / M$ female/male, $F$ Fisher's exact test, $M W$ Mann-Whitney $U$ test, $N S$ not significant, $O C S$ oral corticosteroids, $O T$ olfactory training, $S D$ standard deviation, TDI score threshold-discrimination-identification score

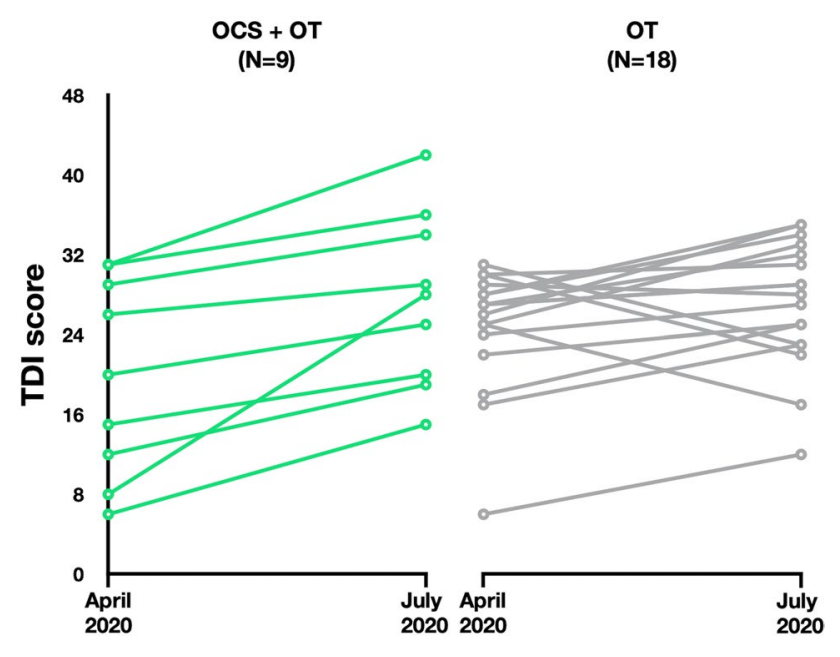

Fig. 1 Evolution of olfactory loss due to COVID-19 for two treatment protocols. Comparison of the evolution of olfactory function (TDI score) between April and July 2020 in dysosmic patients depending on their treatment group: oral corticosteroids and olfactory training $(\mathrm{OCS}+\mathrm{OT} ; n=9$; left) versus olfactory training alone (OT; $n=18$; right). TDI score $=$ threshold - discrimination - identification score

\section{Discussion}

The SARS-CoV-2 pandemic is still ongoing and many patients keep on presenting to medical centers with sudden onset olfactory dysfunction. Although, the majority seems to recover completely after a few weeks, a small proportion seems to display persistent olfactory dysfunction with disturbing symptoms such as parosmia and phantosmia [2]. A small proportion in a global pandemic still represents a colossal number of patients, especially as the number of infected patients continues to grow worldwide. Although typically a very rare complaint in daily ENT practice, permanent olfactory dysfunction may thus become a frequent reason for consultation and solicitation for medical treatment. For this reason, a potential medical treatment may prevent future patients from experiencing a permanent decline in quality of life.

Two recent articles by Miwa et al. and Hura et al. reviewed the literature on medical management for PVOD which included zinc, traditional Japanese medicine, alphalipoic acid, vitamin A, minocycline, theophylline, acupuncture, systemic and topical steroids, and OT [6, 14]. Although none of these treatment options reached grade A evidence of efficacy, both reviews concluded that OT is the recommended approach. OT was associated with minimal harm effect and was found to be of most benefit in improving olfactory function, all the more so if started early on and with high compliance. The only inconvenience was the need for a sustained daily training for months. By contrast, in our study, we did not observe a significant increase in olfactory function in patients performing OT alone after 10 weeks of training [16].

Whether COVID-19-related olfactory dysfunction is the result of excessive inflammation or a sensorineural disorder, or a combination of both is unknown. Similarly, the mechanism by which OT and steroid therapy could reduce the risk of PVOD and improve olfaction is yet to be determined. Studies on dysosmic mice suggest that the decision to treat with steroids or OT may ultimately depend on the cause of the olfactory loss [17]. Recently, MRI studies showed signs of inflammation of the olfactory clefts of COVID-19 patients with acute anosmia compared to healthy controls, suggesting a possible role for antiinflammatory drugs such as steroids $[18,19]$. The idea of treating PVOD with corticosteroids is not new, but highevidence studies are lacking and the few available studies provide conflicting results. As Miwa et al. highlighted, steroid treatment may be effective for post-viral olfactory loss only in the early stages of the disease, when lesions are not yet irreversible. This could explain why patients suffering from PVOD for many years have not responded to steroid therapy. Taken together, both reviews conclude that steroids may be a treatment option if given early on in 
the disease course and in selected patients without medical contra-indications.

In this pilot study, participants in the OCS + OT group showed a significant increase of 7.7 points on average in their TDI score, compared to an insignificant 2.1-increase in the OT group. Moreover, Gudziol et al. showed that the minimal clinically important difference for subjective improvement of smell was a 5.5-point increase in TDI score, suggesting a possible clinical benefit in the OCS + OT group [20]. Besides, as participants started OCS on average 5 weeks after the acute loss of smell, future studies may observe better TDI recovery scores from treating patients during the acute phase of COVID-19. As of today, the use of OCS to treat COVID-19 is still contentious, though significant benefit has been shown on mortality among severe COVID patients with no risk of serious adverse event [10]. With regard to the use of steroids for olfactory dysfunction due to COVID-19, only a case report has been published which endorses the use of oral prednisolone as soon as nasal swab becomes negative [21].

Our study's main limitation was its small sample size, particularly those in the OCS group. This was mainly due to safety concerns surrounding the use of steroids in treating COVID-19 in April 2020. Unfortunately, this prevented us from randomizing our treatment groups. In turn, it is difficult for our preliminary findings to be conclusive about the potential benefits of OCS and OT, and caution is warranted. Ideally, larger cohorts and a control group of dysosmic patients would help separate the effect of treatment from spontaneous recovery. A final limitation was the low level of compliance for olfactory training, which did not exceed $50 \%$ on average in either groups. The fact that only 6 out of 27 patients were anosmic may have limited participants' motivation to perform OT correctly in some hyposmic patients [22]. Moreover, low compliance may partly explain the absence of noticeable improvement in the OT group in our study. Although our statistical tests did not find a correlation between higher compliance to OT and higher TDI score, this is most likely due to the limited group size of our study. Future studies on OT may benefit from higher compliance, longer duration of training, and larger cohorts.

\section{Conclusion}

The findings of this pilot study support the safety and efficacy of combining oral corticosteroids and olfactory training in the management of olfactory dysfunction resulting from COVID-19 infection as well as give impetus for randomized clinical trials to corroborate these results. Future studies on olfactory loss due to COVID-19 may investigate the use of steroids in larger cohorts and earlier in the course of the disease now that safety concerns about corticosteroids may be less founded than previously thought and patients more inclined to take them.

Acknowledgements We thank Gaëlle Calberson, Sylvie Desmet, Marine Dawirs, Danielle Degreef, Ikram Laghmari, Stéphanie Negele and Justine Verbeke for their excellent work in coordinating the study. Thanks to Abdi Mohamed for proofreading the manuscript.

Author contributions LB had full access to all the data in the study and takes responsibility for the integrity of the data and the accuracy of the data analysis. Conceptualization and Methodology: LB, H. Acquisition, analysis, or interpretation of data: LB, H, PI, PR. Original draft preparation: $\mathrm{LB}$. Critical revision of the manuscript for important intellectual content: H, L, K. Statistical analysis: LB.

Funding This study received Smell Training Kits by Dos Medical-BV (Heteren, Netherlands). Dos Medical-BV had no role in the design and conduct of the study; Collection, management, analysis, and interpretation of the data; Preparation, review, or approval of the manuscript; And decision to submit the manuscript for publication.

\section{Compliance with ethical standards}

Conflict of interests The authors have no conflict of interests to declare.

Ethics approval The study was performed in accordance with the Declaration of Helsinki on Biomedical Studies Involving Human Subjects. The study protocol was approved by the Review Board of CHU SaintPierre, Brussels, Belgium (CHUSP 200422).

Consent to participate Informed written consent was obtained from all participants to participate in the study.

\section{References}

1. Boscolo-Rizzo P, Borsetto D, Fabbris C et al (2020) Evolution of altered sense of smell or taste in patients with mildly symptomatic COVID-19. JAMA Otolaryngol Neck Surg. https://doi. org/10.1001/jamaoto.2020.1379

2. Le Bon S-D, Pisarski N, Verbeke J et al (2020) Psychophysical evaluation of chemosensory functions 5 weeks after olfactory loss due to COVID-19: a prospective cohort study on 72 patients. Eur Arch Oto-Rhino-Laryngol. https://doi.org/10.1007/s00405-02006267-2

3. Vaira LA, Hopkins C, Petrocelli M et al (2020) Smell and taste recovery in coronavirus disease 2019 patients: a 60-day objective and prospective study. J Laryngol Otol. https://doi.org/10.1017/ S0022215120001826

4. Huart C, Philpott C, Konstantinidis I et al (2020) Comparison of COVID-19 and common cold chemosensory dysfunction. Rhinology. https://doi.org/10.4193/Rhin20.251

5. Suzuki M, Saito K, Min W-P et al (2007) Identification of viruses in patients with postviral olfactory dysfunction. Laryngoscope 117:272-277. https://doi.org/10.1097/01.mlg.0000249922.37381 $.1 \mathrm{e}$

6. Hura N, Xie DX, Choby GW et al (2020) Treatment of post-viral olfactory dysfunction: an evidence-based review with recommendations. Int Forum Allergy Rhinol 10:1065-1086. https:// doi.org/10.1002/alr.22624 
7. Pinto JM, Wroblewski KE, Kern DW et al (2014) Olfactory dysfunction predicts 5-year mortality in older adults. PLoS ONE 9(10):e107541. https://doi.org/10.1371/journal.pone.0107541

8. Whitcroft KL, Hummel T (2020) Olfactory dysfunction in COVID-19. JAMA 323:2512. https://doi.org/10.1001/ jama.2020.8391

9. The RECOVERY Collaborative Group (2020) Dexamethasone in hospitalized patients with Covid-19: preliminary report. N Engl J Med NEJMoa2021436

10. Sterne JAC, Murthy S, Diaz JV et al (2020) Association between administration of systemic corticosteroids and mortality among critically Ill patients with COVID-19. JAMA. https://doi. org/10.1001/jama.2020.17023

11. Mattos-Silva P, Felix NS, Silva PL et al (2020) Pros and cons of corticosteroid therapy for COVID-19 patients. Respir Physiol Neurobiol 280:103492. https://doi.org/10.1016/j.resp.2020.10349 2

12. Ikeda K, Sakurada T, Suzaki Y, Takasaka T (1995) Efficacy of systemic corticosteroid treatment for anosmia with nasal and paranasal sinus disease. Rhinology 33:162-165

13. Heilmann S, Huettenbrink K-B, Hummel T (2004) Local and systemic administration of corticosteroids in the treatment of olfactory loss. Am J Rhinol 18:29-33. https://doi.org/10.1177/19458 9240401800107

14. Miwa T, Ikeda K, Ishibashi $\mathrm{T}$ et al (2019) Clinical practice guidelines for the management of olfactory dysfunction: secondary publication. Auris Nasus Larynx 46:653-662. https://doi. org/10.1016/j.anl.2019.04.002

15. Oleszkiewicz A, Schriever VA, Croy I et al (2019) Updated Sniffin' Sticks normative data based on an extended sample of 9139 subjects. Eur Arch Oto-Rhino-Laryngol 276:719-728. https://doi. org/10.1007/s00405-018-5248-1
16. Damm M, Pikart LK, Reimann H et al (2014) Olfactory training is helpful in postinfectious olfactory loss: a randomized, controlled, multicenter study. Laryngoscope 124:826-831. https://doi. org/10.1002/lary.24340

17. Kim B-Y, Park JY, Kim E (2020) Differences in mechanisms of steroid therapy and olfactory training for olfactory loss in mice. Am J Rhinol Allergy 34:810-821. https://doi.org/10.1177/19458 92420930945

18. Altundag A, Yıldırım D, Tekcan Sanli DE et al (2020) Olfactory cleft measurements and COVID-19-related anosmia. Otolaryngol Neck Surg. https://doi.org/10.1177/0194599820965920

19. Eliezer M, Hautefort C (2020) MRI evaluation of the olfactory clefts in patients with SARS-CoV-2 infection revealed an unexpected mechanism for olfactory function loss. Acad Radiol 27:1191. https://doi.org/10.1016/j.acra.2020.05.013

20. Gudziol V, Lötsch J, Hähner A et al (2006) Clinical significance of results from olfactory testing. Laryngoscope 116:1858-1863. https://doi.org/10.1097/01.mlg.0000234915.51189.cb

21. Touisserkani SK, Ayatollahi A (2020) Oral corticosteroid relieves post-COVID-19 anosmia in a 35-year-old patient. Case Rep Otolaryngol 2020:1-2. https://doi.org/10.1155/2020/5892047

22. Saatci O, Altundag A, Duz OA, Hummel T (2020) Olfactory training ball improves adherence and olfactory outcomes in post-infectious olfactory dysfunction. Eur Arch Oto-Rhino-Laryngology 277:2125-2132. https://doi.org/10.1007/s00405-020-05939-3

Publisher's Note Springer Nature remains neutral with regard to jurisdictional claims in published maps and institutional affiliations. 